\title{
Construction of a Predictive Model of Respiratory Endoscopic Intervention in Children with Lobar Pneumonia Caused by Mycoplasma Pneumoniae Infection
}

\author{
Jiahui Zhang
}

Children's Hospital of Soochow University

Yu Ma

Children's Hospital of Soochow University

Zhichao Sun

Children's Hospital of Soochow University

Ting Wang

Children's Hospital of Soochow University

Yongdong Yan

Children's Hospital of Soochow University

Li Huang

Children's Hospital of Soochow University

Zhengrong Chen ( $\nabla$ chen_zheng_rong@163.com )

Children's Hospital of Soochow University

\section{Article}

Keywords: lobar pneumonia caused by MP infection, clinical features, prognosis, risk factorss, predictive model.

Posted Date: June 24th, 2022

DOI: https://doi.org/10.21203/rs.3.rs-1257638/v2

License: (c) (i) This work is licensed under a Creative Commons Attribution 4.0 International License. Read Full License 


\section{Abstract}

Objectives: This study aimed to analyze the clinical features of children with lobar pneumonia caused by Mycoplasma pneumoniae (MP) infection, to provide a prognostic evaluation of interventional bronchoscopy, and to explore the independent risk factors for bronchoscopic intervention in children with lobar pneumonia caused by MP infection. We also aimed to construct an early-warning model of bronchoscopic intervention, so as to provide an objective evaluation tool for clinicians.

Methods: We collected the clinical data from 533 children with lobar pneumonia caused by MP infection. The patients were divided into three groups according to the interventional indications for bronchoscopy and whether they were treated with bronchoscopic intervention, and the clinical features and prognosis of the three groups were compared. Binary logistic regression analysis was performed on the indicators with a significance value of $\mathrm{P}<0.05$, which we retrieved from the comparative analysis between the first two groups, in order to uncover the independent risk factors and regression equations concerning bronchoscopic intervention. The regression coefficient ( $\beta$ ) of our regression model was then used to score related values in the model so as to construct a predictive scoring model of bronchoscopic intervention for the treatment of children with lobar pneumonia caused by MP infection.

Results: Children with lobar pneumonia caused by MP infection who demonstrated absolute indications for bronchoscopy exhibited more severe clinical manifestations, and children without absolute indications for bronchoscopy achieved a better prognosis even without bronchoscopic intervention. To establish our early-warning model of bronchoscopic intervention for children with lobar pneumonia caused by MP infection, we utilized the following indices: a C-reactive protein (CRP) $\geq 20.94 \mathrm{mg} / \mathrm{L}\left(\beta_{1}=\right.$ 2.253) received 3 points; a fever duration before bronchoscopy $\geq 6.5 \mathrm{~d}\left(\beta_{2}=1.424\right)$, lactate dehydrogenase $(\mathrm{LDH}) \geq 461.5 \mathrm{U} / \mathrm{L}\left(\beta_{3}=1.246\right)$, or fever $\left(\beta_{4}=1.223\right)$, each received 2 points; and if complicated by pleural effusion $\left(\beta_{5}=0.841\right), 1$ point, for a total score of 10 points.

Conclusions: When the score for the children with lobar pneumonia caused by MP infection was $\geq 6$, the possibility of bronchoscopic intervention for treatment was higher than $80 \%$. The higher the score, the greater the possibility of bronchoscopic intervention.

\section{Introduction}

MP is the smallest prokaryotic pathogenic microorganism known. It is transmitted through droplets and direct contact and can cause disease in any season of the year (1). The incidence of Mycoplasma pneumoniae pneumonia (MPP) has gradually increased annually, and macrolide antibacterials have been used widely as treatment. However, the incidence of macrolide-resistant Mycoplasma pneumoniae pneumonia (MRMP) is increasing worldwide, severely limiting the treatment options for children with $\operatorname{MPP}(2,3)$. 
The chest imaging findings in MPP vary, and lobar pneumonia caused by MP infection is considered to be one of the clinical features of refractory MPP (4). Respiratory endoscopy equipment and technology have seen progressive improvements in recent years, and endoscopy has gradually become an important treatment modality in treating various respiratory diseases in pediatrics (5).

However, children with lobar pneumonia caused by MP infection manifest clinical symptoms and pulmonary infections that are different from those of adults, and whether they should be actively treated with bronchoscopy requires further investigation. In order to better grasp the indications and contraindications of respiratory endoscopic intervention and to avoid over- or under-treatment, we must acknowledge an urgent need for corresponding early-warning models to standardize the indications of bronchoscopic intervention and to provide clinicians with objective assessment tools.

\section{Materials And Methods}

\subsection{Patients and Data Collection}

We herein selected children with lobar pneumonia due to MP infection as our research subjects, and they were all hospitalized at the Children's Hospital of Soochow University. Our study selection criteria were (1) meeting the diagnosis of MP infection, with serum MP-Immunoglobulin M (IgM) $\geq 1.1 \mathrm{COL}$ and/or nasopharyngeal aspirates (NPAs) and/or BALF MP-DNA, determined by fluorescence quantitative PCR, > $10^{4} \mathrm{copies} / \mathrm{ml}$; and (2) manifestation of respiratory symptoms with or without fever, consistent with the diagnosis of chest imaging of lobar pneumonia (6)-i.e., a chest radiograph or chest CT examination that revealed uniform consolidation of one or more segments/lobes of the lung. Exclusion criteria included individuals with contraindications to fiberoptic bronchoscopy; exhibiting bronchopulmonary dysplasia or malformations, repeated respiratory infections, aspiration pneumonia, or exogenous foreign bodies in the bronchus; and those with incomplete medical history data.

The clinical data collected included sex, age, date of hospitalization, pre-hospitalization course and preendoscopic heat course, number of hospitalization days, bronchoscopy time, clinical manifestations and signs, laboratory examinations, and lung imaging examinations.

\subsection{Definitions}

\subsubsection{Classification Criteria}

Our criteria reflected the 'Experts Consensus on Diagnosis and Treatment of Respiratory Endoscopy in Children with Refractory Pneumonia in China' Respiratory Endoscopy Intervention Indications (7) and are as follows. 1. Slow absorption of pneumonia: although the symptoms and signs of the patient improved after treatment with anti-infective drugs, chest X-ray and CT lesion absorption were less than $50 \%$ after two weeks. 2. Pneumonia that persisted for a lengthy time period: the course of the disease was prolonged (> 2 weeks), and the condition was not alleviated after active treatment but deteriorated and 
persisted. 3. Extensive erosion of airway mucosa, necrotic epithelial shedding, mucus plugs blocking the airways, and even bronchial shaping that could be observed under respiratory endoscopy. 4. Imaging showed unilateral emphysema, mediastinal emphysema, disappearance of bronchial inflation signs in unilateral or bilateral lung consolidation, tree bud signs, or other small airway diseases.

\subsubsection{Establishment of Groups According to the Aforementioned Standards}

For the Bronchoscopy Absolute Indication Group (Group A), children with lobar pneumonia caused by MP infection who met any of the four bronchoscopic intervention indications underwent bronchoscopy and lavage during hospitalization; and for the Bronchoscopy Relative Indication Group (Group B), children with lobar pneumonia caused by MP infection underwent bronchoscopy during their hospitalization on the basis of routine anti-infective and anti-inflammatory, cough and phlegm, and atomized inhalation treatments. Thin or flocculent secretions were noted under the bronchoscope, but there were no signs of airway mucosal erosion, necrotic epithelial shedding, mucus plugs blocking the airway, or bronchial shaping. If none of the four indications for bronchoscopic intervention was met, children with lobar pneumonia caused by MP infection were treated with routine anti-infective and anti-inflammatory, cough and phlegm, and atomized inhalation treatments. Bronchoscopic intervention was not implemented during hospitalization (Group C). The three groups comprised 229, 166, and 138 children, respectively. 2.2.3 Prognostic Evaluation Criteria for the Three Groups of Children

Evaluation standards were as follows (8). (1) A normal body temperature: the body temperature was less than $37.4^{\circ} \mathrm{C}$ within $72 \mathrm{~h}$. (2) The cough-degree classifications were as follows: no cough recorded as 0 points; mild cough was an occasional or intermittent cough that did not affect the study or quality of the patient and was recorded as 1 point; moderate cough was between mild and severe and was assigned 2 points; severe cough was frequent or paroxysmal coughing day and night that affected sleep, study, or quality of life and was assigned 3 points. (3) The degree of rales in the lungs was assessed as follows. No rales in either lung was recorded as 0 points; a small amount of rales heard in both lungs was recorded as 1 point; rales that were audible and moderate in both lungs were recorded as 2 points; abundant rales were assigned 3 points. (4) The degree of absorption of lung lesions upon chest X-ray or CT was designated as follows: 0 points for complete absorption of lesions; 1 point for lesion absorption $\geq 1 / 2$; 2 points for lesion absorption $<1 / 2$; and 3 points for no absorption or aggravation of lesions.

Prognostic Evaluation Criteria (8): The children were re-examined using chest radiography or chest CT one week after admission to the hospital; and clinical symptoms, signs, and chest radiographic or chest CT changes were then used as the criteria. (1) For a child to be considered 'cured,' her/his body temperature needed to revert to normal, there was no cough or only a mild cough, lung signs disappeared, and chest X-ray or CT showed that the lung lesions were basically absorbed. (2) 'Markedly effective' was assigned if the child had a normal body temperature, a moderate or lower degree of coughing, if lung signs disappeared or were only slightly present, and if chest X-ray or CT showed that the absorption of lung lesions was $\geq 1 / 2$. (3) An 'effective' rating was delivered if the child's body temperature was normal, the degree of coughing was moderate, the lung signs were alleviated, and the chest radiograph or chest CT indicated that the absorption of lung lesions was less than $1 / 2$. (4) A rating of 'ineffective' indicated that the child's body temperature did not drop significantly, the child showed a degree of moderate or severe 
coughing, both lungs were full of rales or lung rales worse than before, and the chest X-ray or CT showed that the lung lesions were not absorbed or that they had worsened. (5) The total effective rate $=$ (cured + markedly effective + effective cases)/total number of cases $\times 100 \%$.

\subsection{Statistical Analyses}

We used SPSS v. 24.0 statistical software for the data analysis. The measurement data that conformed to a normal distribution are expressed as means \pm standard deviation. Two independent-sample $t$ tests were used for comparisons between two groups, and one-way ANOVA was used to compare three groups. After the Bonferroni method corrected the significance level, a pairwise comparison was performed. The measurement data that did not conform to a normal distribution are presented as the median (25th percentile, 75th percentile), and we used the Mann-Whitney $U$ test to compare two groups and the KruskalWallis $\mathrm{H}$ test for three groups. The Bonferroni method was then used for comparisons between groups. Counting data are expressed as percentages (\%), with comparisons between groups executed with the $\chi^{2}$ test and the Fisher exact-probability method depending upon which conditions were met. The differences between groups were statistically significant at $P<0.05$. We used a binary logistic regression analysis (backward stepwise method) to establish a regression equation model to obtain the probability of bronchoscopic intervention for children with lobar pneumonia and drew a receiver operating characteristic (ROC) curve. Based on the $\beta$ coefficient in the binary logistic regression model, the factors in the model were weighted to construct a predictive scoring system for children with lobar pneumonia caused by MP infection that required bronchoscopic intervention.

\section{Results}

\subsection{Clinical Characteristics}

We enrolled a total of 533 children who met the inclusion criteria. In group A, there were 229 casesincluding 126 males (55.0\%) and 103 females (45.0\%)-and the male-to-female ratio was $1.22: 1$, with a median age of 5.92 years. In group B, there were 166 cases-including 82 males (49.4\%) and 84 females (50.6\%) -and the male-to-female ratio was $0.97: 1$, with a median age of 5.67 years. In group C, there were 138 cases-including 69 males (50.0\%) and 69 females (50.0\%)-and the male-to-female ratio was 1:1, with a median age of 5.83 years. There were no significant differences in either the average age or sex in the three groups of children $(P>0.05$, Table 1$)$. There were also no significant differences in the incidence rates of wheezing history, eczema history, allergic rhinitis history, or asthma history among the three groups of children ( $12.66 \%$ vs. $15.06 \%$ vs. $16.67 \%$; $34.50 \%$ vs. $35.54 \%$ vs. $36.96 \% ; 14.85 \%$ vs. $15.06 \%$ vs. $18.12 \% ; 1.31 \%$ vs. $4.22 \%$ vs. $1.45 \%$; respectively; $P>0.05$, Table 1$)$. The ratio of disease duration prior to admission, fever duration before bronchoscopy, and fever peak in group A were all higher than in the latter two groups $(P<0.05$, Table 1$)$. 
Table 1

Comparison of clinical characteristics among the three groups of children.

\begin{tabular}{|c|c|c|c|c|}
\hline Variables & $\begin{array}{l}\text { Group A (n } \\
=229)\end{array}$ & $\begin{array}{l}\text { Group B } \\
(n=166)\end{array}$ & $\begin{array}{l}\text { Group C } \\
(n=138)\end{array}$ & Statistics \\
\hline
\end{tabular}

Characteristics

\begin{tabular}{|c|c|c|c|c|c|}
\hline Sex (male/female, $n$ ) & $126 / 103$ & $82 / 84$ & $69 / 69$ & $1.509 \Delta$ & 0.470 \\
\hline Age M (P25-P75)/year & $\begin{array}{l}5.92(3.67, \\
7.58)\end{array}$ & $\begin{array}{l}5.67(3.67, \\
7.67)\end{array}$ & $\begin{array}{l}5.83(3.83 \\
6.83)\end{array}$ & $1.192^{\square}$ & 0.551 \\
\hline Wheezing history, n (\%) & $29(12.66)$ & $25(15.06)$ & $23(16.67)$ & $1.190^{\Delta}$ & 0.552 \\
\hline Eczema history, n (\%) & 79 (34.50) & 59 (35.54) & $51(36.96)$ & $0.228^{\Delta}$ & 0.892 \\
\hline Allergic rhinitis history, n (\%) & $34(14.85)$ & $25(15.06)$ & $25(18.12)$ & $0.782^{\Delta}$ & 0.676 \\
\hline Asthma history, n (\%) & $3(1.31)$ & $7(4.22)$ & $2(1.45)$ & $4.239 \Delta$ & 0.120 \\
\hline $\begin{array}{l}\text { Disease duration before } \\
\text { admission, M (P25-P75)/d }\end{array}$ & $7(6,10)$ a,b & $7(5,10)$ & $7(5,9)$ & $9.929^{\square}$ & 0.007 \\
\hline $\begin{array}{l}\text { Fever duration before } \\
\text { bronchoscopy M (P25-P75)/d }\end{array}$ & $7(5,10)^{a, b}$ & $5(0,7)$ & $5(4,7)$ & $42.359^{\square}$ & 0.000 \\
\hline Hot peak M (P25-P75), ${ }^{\circ} \mathrm{C}$ & $\begin{array}{l}39.6(39.0 \\
40.0)^{a, b}\end{array}$ & $\begin{array}{l}39(38.5 \\
39.7)\end{array}$ & $\begin{array}{l}39(38.7 \\
39.5)\end{array}$ & $45.561^{\square}$ & 0.000 \\
\hline
\end{tabular}

Signs and symptoms, $\mathrm{n}(\%)$

\begin{tabular}{|lccccc|}
\hline Fever & $219(95.6)^{a}$ & $143(86.1)$ & $126(91.3)$ & $11.225^{\Delta}$ & 0.004 \\
\hline Cough & $228(99.6)$ & $166(100)$ & $138(100)$ & $1.330^{\Delta}$ & 0.514 \\
\hline Respite & $24(10.48)$ & $14(8.43)$ & $17(12.32)$ & $1.240 \Delta$ & 0.538 \\
\hline Shortness of breath & $13(5.68)$ & $8(4.82)$ & $5(3.62)$ & $0.784 \Delta$ & 0.676 \\
\hline $\begin{array}{l}\text { Difficulty breathing } \\
\text { Nasal congestion and runny } \\
\text { nose }\end{array}$ & $5(2.18)$ & $3(1.81)$ & $2(1.45)$ & $0.258 \Delta$ & 0.879 \\
\hline $\begin{array}{l}\text { Pulmonary rales } \\
\text { (1) }\end{array}$ & $102(44.5)$ & $34(20.48)$ & $31(22.46)$ & $1.177 \Delta$ & 0.555 \\
\hline
\end{tabular}

Note: Group A, bronchoscopic absolute indication group; Group $B$, bronchoscopic relative indication group; Group $C$, bronchoscopic intervention was not provided during hospitalization. $\Delta, \chi^{2}$ value obtained by Pearson test; , $H$ value obtained by Kruskal-Wallis $H$ test; and after the significance level was corrected with the Bonferroni method, pairwise comparisons were performed. a, $P<0.05$ after correction compared with groups $A$ and $B ; b, P<0.05$ after correction compared between groups $A$ and C. 


\begin{tabular}{|c|c|c|c|c|c|}
\hline Variables & $\begin{array}{l}\text { Group A ( } n \\
=229)\end{array}$ & $\begin{array}{l}\text { Group B } \\
(n=166)\end{array}$ & $\begin{array}{l}\text { Group C } \\
(n=138)\end{array}$ & Statistics & $P$ value \\
\hline Decreased breath sounds & $64(27.9)^{b}$ & $31(18.7)$ & $15(10.9)$ & $15.901 \Delta$ & 0.000 \\
\hline \multicolumn{6}{|c|}{$\begin{array}{l}\text { Note: Group A, bronchoscopic absolute indication group; Group } B, \text { bronchoscopic relative indication } \\
\text { group; Group } C \text {, bronchoscopic intervention was not provided during hospitalization. } \Delta, \chi^{2} \text { value } \\
\text { obtained by Pearson test; }, H \text { value obtained by Kruskal-Wallis } H \text { test; and after the significance level } \\
\text { was corrected with the Bonferroni method, pairwise comparisons were performed. a, } P<0.05 \text { after } \\
\text { correction compared with groups } A \text { and } B ; b, P<0.05 \text { after correction compared between groups } A \text { ana } \\
C \text {. }\end{array}$} \\
\hline
\end{tabular}

White blood cell count (WBC), neutrophil percentage (N\%), C-reactive protein (CRP), lactate dehydrogenase (LDH), alanine aminotransferase (ALT), aspartate aminotransferase (AST), total value of Immunoglobulin A ( $\operatorname{IgA})$, complications with pulmonary atelectasis, and complications with pleural effusion in group $A$ were all higher than in the latter two groups $(P<0.05$, Table 2$)$. MP-DNA copy numbers (medium- and high-load groups) in group $A$ were higher than in the latter two groups $(P<0.05$, Fig. 1), while the percentages of $\mathrm{CD} 3^{+} \mathrm{CD} 4^{+}$and $\mathrm{CD} 4^{+} / \mathrm{CD} 8^{+}$cells in lymphocyte subpopulations were lower than in the latter two groups $(P<0.05$, Table 2). The level of prealbumin $(P A)$ in group $A$ was also significantly lower than that in group $B$ (after correction, $P<0.05$, Table 2 ). 
Table 2

Comparison of laboratory indices and imaging characteristics among the three groups of children.

\begin{tabular}{|c|c|c|c|c|c|}
\hline Variables & $\begin{array}{l}\text { Group } A(n= \\
229)\end{array}$ & $\begin{array}{l}\text { Group } B(n= \\
166)\end{array}$ & $\begin{array}{l}\text { Group } C(n= \\
138)\end{array}$ & Statistics & $\begin{array}{l}P \\
\text { value }\end{array}$ \\
\hline $\begin{array}{l}\text { WBC, M (P25-P75)/ } \\
\times 10^{9} \mathrm{~L}^{-1}\end{array}$ & $\begin{array}{l}8.97(6.78, \\
12.20)^{a, b}\end{array}$ & $\begin{array}{l}7.59 \\
(6.05,10.57)\end{array}$ & $7.00(5.73,9.63)$ & 20.253 & 0.000 \\
\hline N, M (P25-P75)/\% & $\begin{array}{l}67.40(56.85 \\
76.7)^{a, b}\end{array}$ & $\begin{array}{l}55.05(44.02 \\
65.93)^{c}\end{array}$ & $\begin{array}{l}59.00(44.0 \\
68.28)\end{array}$ & 60.368 & 0.000 \\
\hline $\begin{array}{l}\text { EOS, M (P25-P75)/ } \\
\times 10^{9} \mathrm{~L}^{-1}\end{array}$ & $0.20(0.01,0.85)$ & $0.40(0.07,1.30)$ & $0.50(0.10,1.10)$ & 5.473 & 0.065 \\
\hline $\begin{array}{l}\text { CRP, M (P25- } \\
\mathrm{P} 75) / \mathrm{mg}^{-1} \mathrm{~L}^{-1}\end{array}$ & $\begin{array}{l}27.37 \\
(14.55,49.29)^{a, b}\end{array}$ & $\begin{array}{l}6.63 \\
(1.62,16.42)\end{array}$ & $\begin{array}{l}8.13 \\
(3.69,18.90)\end{array}$ & 120.547 & 0.000 \\
\hline $\begin{array}{l}\text { PLT, M (P25- } \\
\text { P75) } \times 10^{9} \mathrm{~L}^{-1}\end{array}$ & $307(226,394)$ & $\begin{array}{l}300.50 \\
(239.50,406.50)\end{array}$ & $286(234,383)$ & 0.772 & 0.680 \\
\hline $\begin{array}{l}\text { LDH, M (P25- } \\
\text { P75)/U.L }{ }^{-1}\end{array}$ & $\begin{array}{l}474.80 \\
(366.15,611.50) \\
a, b\end{array}$ & $\begin{array}{l}357.15 \\
(309.97,424.30)\end{array}$ & $\begin{array}{l}333.10 \\
(294.95,415.45)\end{array}$ & 85.557 & 0.000 \\
\hline $\begin{array}{l}\text { PA, M (P25- } \\
\text { P75)/mg.L }{ }^{-1}\end{array}$ & $\begin{array}{l}132 \\
(108.5,163.5)^{a}\end{array}$ & $\begin{array}{l}150.5 \\
(121.75,203.75) \\
c\end{array}$ & $\begin{array}{l}138.00 \\
(122.50,158.25)\end{array}$ & 24.492 & 0.000 \\
\hline $\begin{array}{l}\text { ALT, M (P25-P75) } \\
/ U \cdot L^{-1}\end{array}$ & $\begin{array}{l}15 \cdot 30 \\
(11.16,26.65)^{a, b}\end{array}$ & $\begin{array}{l}13.00 \\
(9.98,17.08)\end{array}$ & $\begin{array}{l}12.00 \\
(10.18,15.53)\end{array}$ & 25.391 & 0.000 \\
\hline $\begin{array}{l}\text { AST, M (P25- } \\
\text { P75)]/U. } L^{-1}\end{array}$ & $\begin{array}{l}32.50 \\
(25.95,43.50)^{a, b}\end{array}$ & $\begin{array}{l}28.55 \\
(23.68,35.25)\end{array}$ & $\begin{array}{l}29.15 \\
(24.90,34.53)\end{array}$ & 17.235 & 0.000 \\
\hline $\begin{array}{l}\text { Serum MP-IgM M } \\
\text { (P25-P75) /COL }\end{array}$ & $\begin{array}{l}2.96(0.63,5.99) \\
b\end{array}$ & $\begin{array}{l}2.65(0.60,5.86) \\
c\end{array}$ & $1.41(0.39,3.86)$ & 15.300 & 0.000 \\
\hline $\begin{array}{l}\text { Serum MP-lgG, M } \\
\text { (P25-P75) /COL }\end{array}$ & $\begin{array}{l}20.33 \\
(3.12,189.31)\end{array}$ & $\begin{array}{l}6.71 \\
(2.00,100.80)\end{array}$ & $\begin{array}{l}15.56 \\
(2.96,122.18)\end{array}$ & 4.132 & 0.127 \\
\hline $\begin{array}{l}\text { Sputum MP-DNA } \\
\text { copy number, n (\%) }\end{array}$ & & & & & \\
\hline
\end{tabular}

Note: Group A, bronchoscopic absolute indication group; Group B, bronchoscopic relative indication group; Group $C$, bronchoscopic intervention was not provided during hospitalization. $\Delta, \chi^{2}$ value obtained from Pearson test; , $H$ value obtained from Kruskal-Wallis $H$ test; $\boldsymbol{\Lambda}, F$ value obtained from one-way ANOVA, with post-hoc pairwise comparisons made after the Bonferroni method was used to correct the significance level. a, $P<0.05$ after correction compared with groups $A$ and $B ; b, P<0.05$ after correction compared between groups $A$ and $C ; c, P<0.05$ after correction compared between groups $B$ and $C$. The copy number was divided into three groups according to the MP-DNA of the child's sputum specimen: low-copy group, MP-DNA copy number $\leq 10^{4} / \mathrm{mL}$; medium-copy group, MPDNA copy number $10^{4}-10^{6} / \mathrm{mL}$; high-copy group, MP-DNA copy number $>10^{6} / \mathrm{mL}$. 


\begin{tabular}{|c|c|c|c|c|c|}
\hline Variables & $\begin{array}{l}\text { Group } A(n= \\
229)\end{array}$ & $\begin{array}{l}\text { Group } B(n= \\
166)\end{array}$ & $\begin{array}{l}\text { Group } C(n= \\
138)\end{array}$ & Statistics & $\begin{array}{l}P \\
\text { value }\end{array}$ \\
\hline Low-load group & $37(16.2)$ & $23(13.86)$ & $18(13.04)$ & $0.785^{\triangle}$ & 0.675 \\
\hline Medium-load group & $25(10.9)^{a, b}$ & $49(29.52)$ & $44(26.51)$ & $29.577 \triangle$ & 0.000 \\
\hline High-load group & $167(72.9)^{a, b}$ & $94(56.62)$ & $76(55.07)$ & $16.322 \triangle$ & 0.000 \\
\hline \multicolumn{6}{|l|}{$\begin{array}{l}\text { Humoral immunity } \\
M(P 25-P 75) / g \cdot L^{-1}\end{array}$} \\
\hline $\lg G$ & $\begin{array}{l}9.28 \\
(7.80,11.09)\end{array}$ & $\begin{array}{l}9.23 \\
(7.40,10.88)\end{array}$ & $\begin{array}{l}9.45 \\
(7.57,10.93)\end{array}$ & 1.126 & 0.570 \\
\hline $\lg A$ & $\begin{array}{l}1.21(0.84,1.63) \\
a, b\end{array}$ & $1.05(0.64,1.44)$ & $0.98(0.57,1.37)$ & 13.084 & 0.001 \\
\hline $\operatorname{lgM}$ & $1.47(1.10,2.17)$ & $1.51(1.07,1.89)$ & $1.36(1.05,1.80)$ & 6.000 & 0.050 \\
\hline \multicolumn{6}{|l|}{ Cellular immunity } \\
\hline $\begin{array}{l}\mathrm{CD}^{+} \mathrm{T}, \mathrm{M}(\mathrm{P} 25- \\
\mathrm{P} 75)] / \%\end{array}$ & $64.4(55.6,72.6)$ & $\begin{array}{l}66.85 \\
(60.28,72.30)\end{array}$ & $\begin{array}{l}65.85 \\
(60.20,71.18)\end{array}$ & 2.970 & 0.226 \\
\hline $\begin{array}{l}\mathrm{CD}^{+}{ }^{+} \mathrm{CD} 4^{+} \mathrm{T},(-\mathrm{x} \pm \\
\mathrm{s}) / \%\end{array}$ & $33.22 \pm 9.27^{a, b}$ & $35.81 \pm 8.37$ & $35.44 \pm 8.09$ & $5.136^{\Delta}$ & 0.006 \\
\hline $\begin{array}{l}\mathrm{CD} 3^{+} \mathrm{CD}^{+} \mathrm{T},(-\mathrm{x} \pm \\
\text { s) } / \%\end{array}$ & $27.88 \pm 7.94$ & $26.53 \pm 7.11$ & $26.06 \pm 6.62$ & $3.076^{\Delta}$ & 0.047 \\
\hline $\begin{array}{l}\mathrm{CD}^{+} / \mathrm{CD}^{+} \mathrm{T}, \mathrm{M} \\
(\mathrm{P} 25-\mathrm{P} 75) / \%\end{array}$ & $\begin{array}{l}1.20 \\
a, b\end{array}(0.90,1.50)$ & $1.3(1.0,1.8)$ & $1.4(1.0,1.8)$ & 14.043 & 0.001 \\
\hline $\begin{array}{l}\text { CD3-CD }(15+56)^{+} \mathrm{T} \\
\mathrm{M}(\mathrm{P} 25-\mathrm{P} 75) / \%\end{array}$ & $\begin{array}{l}9.70 \\
(5.75,15.80)\end{array}$ & $10.2(6.4,15.8)$ & $9.40(7.08,14.7)$ & 0.777 & 0.678 \\
\hline $\begin{array}{l}\mathrm{CD}^{-}{ }^{-} \mathrm{CD} 19^{+} \mathrm{T}, \mathrm{M} \\
(\mathrm{P} 25-\mathrm{P} 75) / \%\end{array}$ & $\begin{array}{l}20.80 \\
(14.20,28.55)\end{array}$ & $\begin{array}{l}20.20 \\
(13.95,25.93)\end{array}$ & $\begin{array}{l}21.45 \\
(16.05,27.68)\end{array}$ & $2.260^{\square}$ & 0.323 \\
\hline $\begin{array}{l}\mathrm{CD} 19^{+} \mathrm{CD} 23^{+} \mathrm{T}, \mathrm{M} \\
(\mathrm{P} 25-\mathrm{P} 75) / \%\end{array}$ & $7.3(5.0,10.70)^{b}$ & $\begin{array}{l}8.00 \\
(5.87,11.35)\end{array}$ & $\begin{array}{l}8.85 \\
(5.55,13.10)\end{array}$ & 8.024 & 0.018 \\
\hline
\end{tabular}

Note: Group A, bronchoscopic absolute indication group; Group $B$, bronchoscopic relative indication group; Group $C$, bronchoscopic intervention was not provided during hospitalization. $\Delta, \chi^{2}$ value obtained from Pearson test; , $H$ value obtained from Kruskal-Wallis H test; $\boldsymbol{\Lambda}, F$ value obtained from one-way ANOVA, with post-hoc pairwise comparisons made after the Bonferroni method was used to correct the significance level. a, $P<0.05$ after correction compared with groups $A$ and $B ; b, P<0.05$ after correction compared between groups $A$ and $C ; C, P<0.05$ after correction compared between groups $B$ and $C$. The copy number was divided into three groups according to the MP-DNA of the child's sputum specimen: low-copy group, MP-DNA copy number $\leq 10^{4} / \mathrm{mL}$; medium-copy group, MPDNA copy number $10^{4}-10^{6} / \mathrm{mL}$; high-copy group, MP-DNA copy number $>10^{6} / \mathrm{mL}$. 


\begin{tabular}{|c|c|c|c|c|c|}
\hline Variables & $\begin{array}{l}\text { Group } A(n= \\
229)\end{array}$ & $\begin{array}{l}\text { Group } B(n= \\
166)\end{array}$ & $\begin{array}{l}\text { Group } C(n= \\
138)\end{array}$ & Statistics & $\begin{array}{l}P \\
\text { value }\end{array}$ \\
\hline \multicolumn{6}{|l|}{$\begin{array}{l}\text { Imaging } \\
\text { characteristics }\end{array}$} \\
\hline $\begin{array}{l}\text { Left-side pneumonia, } \\
n(\%)\end{array}$ & $60(26.20)$ & $60(36.15)$ & $50(36.23)$ & $5.993^{\triangle}$ & 0.050 \\
\hline $\begin{array}{l}\text { Right-side } \\
\text { pneumonia, n (\%) }\end{array}$ & $101(44.10)$ & 72 (43.37) & 59 (42.76) & $0.066^{\triangle}$ & 0.967 \\
\hline $\begin{array}{l}\text { Inflammation on } \\
\text { both sides, } \mathrm{n}(\%)\end{array}$ & 68 (29.70) & 34 (20.48) & $29(21.01)$ & $5.682^{\triangle}$ & 0.058 \\
\hline $\begin{array}{l}\text { Pulmonary } \\
\text { atelectasis, n (\%) }\end{array}$ & $25(10.92)^{a, b}$ & $5(3.01)$ & $2(1.45)$ & $17.501^{\triangle}$ & 0.000 \\
\hline $\begin{array}{l}\text { Pleural effusion, } \mathrm{n} \\
(\%)\end{array}$ & $56(24.45)^{a, b}$ & $16(9.63)$ & $13(9.42)$ & $21.678^{\triangle}$ & 0.000 \\
\hline \multicolumn{6}{|l|}{$\begin{array}{l}\text { Pleural effusion site, } \\
\text { n (\%) }\end{array}$} \\
\hline Left side & $17(7.42)^{b}$ & $9(5.42)$ & $3(2.17)$ & $13.622^{\triangle}$ & 0.001 \\
\hline Right side & $33(14.41)^{a}$ & $5(3.01)$ & $10(7.25)$ & $15.962^{\triangle}$ & 0.000 \\
\hline Both sides & $6(2.62)$ & $2(1.20)$ & $0(0)$ & $4.141^{\triangle}$ & 0.126 \\
\hline \multicolumn{6}{|c|}{$\begin{array}{l}\text { Note: Group A, bronchoscopic absolute indication group; Group } B \text {, bronchoscopic relative indication } \\
\text { group; Group C, bronchoscopic intervention was not provided during hospitalization. } \triangle, \chi^{2} \text { value } \\
\text { obtained from Pearson test; }, H \text { value obtained from Kruskal-Wallis } H \text { test; } \boldsymbol{A} \text {, } F \text { value obtained from } \\
\text { one-way ANOVA, with post-hoc pairwise comparisons made after the Bonferroni method was used to } \\
\text { correct the significance level. a, } P<0.05 \text { after correction compared with groups } A \text { and } B ; b, P<0.05 \\
\text { after correction compared between groups } A \text { and } C ; C, P<0.05 \text { after correction compared between } \\
\text { groups } B \text { and C. The copy number was divided into three groups according to the MP-DNA of the } \\
\text { child's sputum specimen: low-copy group, MP-DNA copy number } \leq 10^{4} / \mathrm{mL} ; \text { medium-copy group, MP- } \\
\text { DNA copy number } 10^{4}-10^{6} / \mathrm{mL} \text {; high-copy group, MP-DNA copy number }>10^{6} / \mathrm{mL} \text {. }\end{array}$} \\
\hline
\end{tabular}

\section{Abbreviations}

MPP: Mycoplasma Pneumoniae Pneumonia WBC: White blood cells;ALT: Alanine aminotransferase; AST: Aspartate amino transferase; CAP: Community-acquired pneumonia; MRMP macrolide-resistant Mycoplasma pneumoniae CARDS Tx community-acquired respiratory distress syndrometoxin BALF Bronchoalveolar lavage fluid NPA nasopharyngeal aspirates CD: Cluster of differentiation; Cl: Confidence interval; CRP: C reactive protein; CS: Corticosteroid;CT: Computed tomography; DD: D-dimer; EOS: Eosinophil count,IgA: Immunoglobulin A; IgG: Immunoglobulin G;LDH: Lactate dehydrogenase; NPA: Nasopharyngeal aspirates; OR: Odds ratio; ROC: Receiver operating characteristic; AUC: Areaunder curve; BMP: Bronchial mucus plugs; 


\subsection{Evaluation of the Prognosis for the Three Groups of Children One Week After Admission to the Hospital}

The median hospitalization time for children in group $A$ was 10 days, which was longer than the 8 days in group $B$ and 7 days in group $C$ (after correction, all $P<0.05$ ), and that of group $B$ was longer than that of group $C$ (after correction, $P<0.05$,). The fever subsidence time in group $A$ was longer than in the latter two groups (after correction, all $\mathrm{P}<0.05$ ). On the 7 th day of hospitalization, the scores for the degrees of coughing, moist pulmonary rales, and lesion absorption in group A were $1(1,2), 1(0,1)$, and $2(2,3)$, respectively; the scores in group $B$ were $1(1,1), 0(0,1)$, and $1(1,2)$, respectively; and in group $C$, the scores were $1(1,1), 0(0,1)$, and $1(1,2)$, respectively-with the scores in group $A$ being higher than those in groups $B$ and $C$. The baseline levels for children in groups $B$ and $C$ were the same. We observed no differences in hospitalization time, fever subsidence time, the scores for degrees of coughing, moist pulmonary rales, or lesion absorption one week after hospitalization between the two groups (after correction, all $\mathrm{P}>0.05$ ).

When we compared the total effective rate and cure rate among the three groups after one week of hospitalization and treatment, we observed that for group A, the total number of effective cases was 155 (67.69\%)-including 0 cases (0) cured, 31 cases (13.54\%) markedly effective, 124 cases $(54.15 \%)$ effective, and 74 cases (32.31\%) ineffective. In group B, the total effective cases were $128(77.11 \%)-$ including 17 cases cured (10.24\%), 66 cases markedly effective (39.76\%), 45 cases effective $(27.10 \%)$, and 38 cases ineffective (22.90\%). In group C, the total effective cases were 116 (84.06\%)-including 17 cases cured (12.32\%), 57 cases markedly effective (41.31\%), 42 cases effective (30.43\%), and 22 cases ineffective (15.94\%). There were no significant differences in the total effective rate or cure rate between groups $B$ and $C$ (after correction, all $P>0.05$ ).

\subsection{Independent Risk Factors for Bronchoscopic Intervention in Children with Lobar Pneumonia Caused by MP Infection}

The indicators with a $\mathrm{P}<0.05$ between groups $\mathrm{A}$ and $\mathrm{B}$ were analyzed by backward stepwise binary logistic regression analysis, and our results showed that fever duration before bronchoscopy (odds ratio [OR], 1.180; 95\% confidence interval [Cl], 1.093-1.275), CRP (OR, 1.048; 95\% Cl, 1.028-1.067), LDH (OR, $1.004 ; 95 \% \mathrm{Cl}, 1.002-1.006)$, fever (OR, 4.609; $95 \% \mathrm{Cl}, 1.533-13.851]$, and complication with pleural effusion (OR, 3.954; $95 \% \mathrm{Cl}, 1.845-8.473$ ] were independent risk factors for bronchoscopic intervention in children with lobar pneumonia caused by MP infection (Table 3). The abovementioned five variables were included in the best regression equation, and the regression equation for predictive probability was logit $(P)=-7.532+0.166 X_{1}+0.047 X_{2}+0.004 X_{3}+1.528 X_{4}+1.375 X_{5}$. (Note: $X_{n}$, the independent variable; $X_{1}$, fever duration before bronchoscopy; $X_{2}, \mathrm{CRP} ; \mathrm{X}_{3}, \mathrm{LDH} ; \mathrm{X}_{4}$, fever; $\mathrm{X}_{5}$, pleural effusion.) 
Table 3

Logistic regression analysis of risk factors related to lobar pneumonia caused by MP infection that requires bronchoscopy.

\begin{tabular}{|c|c|c|c|c|c|}
\hline Variable & $\begin{array}{l}\text { Partial regression } \\
\text { coefficient }(\beta)\end{array}$ & SE & $\begin{array}{l}\text { Wald } \chi 2 \\
\text { value }\end{array}$ & $\begin{array}{l}P \\
\text { value }\end{array}$ & OR $(95 \% \mathrm{Cl})$ \\
\hline $\begin{array}{l}\text { Fever duration before } \\
\text { bronchoscopy (d) }\end{array}$ & 0.166 & 0.039 & 17.745 & 0.000 & $\begin{array}{l}1.180 \\
(1.093- \\
1.275)\end{array}$ \\
\hline $\mathrm{CRP}(\mathrm{mg} / \mathrm{L})$ & 0.047 & 0.009 & 24.264 & 0.000 & $\begin{array}{l}1.048 \\
(1.028- \\
1.067)\end{array}$ \\
\hline $\mathrm{LDH}(\mathrm{U} / \mathrm{L})$ & 0.004 & 0.001 & 14.074 & 0.000 & $\begin{array}{l}1.004 \\
(1.002- \\
1.006)\end{array}$ \\
\hline Fever & 1.528 & 0.561 & 7.406 & 0.007 & $\begin{array}{l}4.609 \\
(1.533- \\
13.851)\end{array}$ \\
\hline Pleural effusion & 1.375 & 0.389 & 12.497 & 0.000 & $\begin{array}{l}3.954 \\
(1.845- \\
8.473)\end{array}$ \\
\hline constant & -7.531 & 1.206 & 38.986 & 0.000 & -- \\
\hline
\end{tabular}

The Predictive Value of Independent Risk Factors in Children with Lobar Pneumonia Caused by MP Infection Who Require Bronchoscopy

The critical values for the independent factors were used to assign the values for each factor. The fever duration before bronchoscopy $\geq 6.5 \mathrm{~d}, \mathrm{CRP} \geq 20.94 \mathrm{mg} / \mathrm{L}, \mathrm{LDH} \geq 461.5 \mathrm{U} / \mathrm{L}$, fever, and complication with pleural effusion were the risk factors for bronchoscopic intervention in children with MP-infected lobar pneumonia; and the logistic regression analysis after assigning values to each risk factor was statistically significant $(P<0.05$, Table 4$)$. The ROC curve was plotted with the predicted probability value of the regression model, and the AUC was $0.860(95 \% \mathrm{Cl}, 0.824-0.897 ; \mathrm{P}=0.000)$, indicating that the predicted probability model had an upper-middle diagnostic accuracy (Fig. 1). 
Table 4

Logistic regression analysis after the assignment of each independent risk factor.

\begin{tabular}{|c|c|c|c|c|}
\hline Variable & $\begin{array}{l}\text { Partial regression } \\
\text { coefficient }(\beta)\end{array}$ & $\begin{array}{l}\text { Wald } \chi^{2} \\
\text { value }\end{array}$ & $\begin{array}{l}P \\
\text { value }\end{array}$ & OR $(95 \% \mathrm{Cl})$ \\
\hline $\begin{array}{l}\text { Fever duration before bronchoscopy }(< \\
6.5 \mathrm{~d}=0 ; \geq 6.5 \mathrm{~d}=1)\end{array}$ & 1.424 & 27.507 & 0.000 & $\begin{array}{l}4.155 \\
(2.440- \\
7.074)\end{array}$ \\
\hline $\begin{array}{l}\mathrm{CRP}(<20.94 \mathrm{mg} / \mathrm{L}=0 ; \geq 20.94 \mathrm{mg} / \mathrm{L}= \\
\text { 1) }\end{array}$ & 2.253 & 60.055 & 0.000 & $\begin{array}{l}9.513 \\
(5.381- \\
16.816)\end{array}$ \\
\hline $\mathrm{LDH}(<461.5 \mathrm{U} / \mathrm{L}=0 ; \geq 461.5 \mathrm{U} / \mathrm{L}=1)$ & 1.246 & 18.292 & 0.000 & $\begin{array}{l}3.476 \\
(1.964- \\
6.153)\end{array}$ \\
\hline Fever $($ no $=0 ;$ yes $=1)$ & 1.223 & 5.756 & 0.016 & $\begin{array}{l}3.397 \\
(1.251- \\
9.228)\end{array}$ \\
\hline Pleural effusion (no $=0$; yes $=1$ ) & 0.841 & 5.325 & 0.021 & $\begin{array}{l}2.319 \\
(1.135- \\
4.738)\end{array}$ \\
\hline
\end{tabular}

Establishment of a Scoring System for Bronchoscopic Intervention in Children with Lobar Pneumonia Caused by MP Infection

According to the $\beta$ value, each independent risk factor was scored separately. CRP $\geq 20.94 \mathrm{mg} / \mathrm{L}$ received 3 points $\left(\beta_{1}=1.424\right)$, fever duration before bronchoscopy $\geq 6.5 \mathrm{~d}\left(\beta_{2}=2.253\right) 2$ points, $L D H \geq 461.5 \mathrm{U} / \mathrm{L}$ $\left(\beta_{3}=1.246\right) 2$ points, fever $\left(\beta_{4}=1.223\right) 2$ points, and complication with pleural effusion $\left(\beta_{5}=0.841\right)$ received 1 point; such that the total score was 10 points (Table 5 ). Using the scores for risk factors to evaluate the risk of bronchoscopic intervention as treatment for children with lobar pneumonia caused by MP infection, we calculated a predictive AUC score of $0.859(95 \% \mathrm{Cl}, 0.821-0.896)$ (Fig. 1). 
Table 5

Assignment table of risk factors.

\begin{tabular}{|c|c|c|c|c|c|}
\hline Factors & Category & $\begin{array}{l}\text { Reference } \\
\left(\mathrm{W}_{\mathrm{ij}}\right)\end{array}$ & $\begin{array}{l}\text { Regression } \\
\text { coefficients }\left(\beta_{i}\right)\end{array}$ & $\begin{array}{l}\beta_{\mathrm{i}}\left(\mathrm{W}_{\mathrm{ij}}\right. \\
\left.\overline{\mathrm{W}}_{\mathrm{iREF}}\right)\end{array}$ & $\begin{array}{l}\text { Point }_{i j}=\beta_{i}\left(W_{i j}\right. \\
\left.-W_{i R E F}\right) / B\end{array}$ \\
\hline \multirow{2}{*}{$\begin{array}{l}\text { Fever duration before } \\
\text { bronchoscopy } \geq 6.5 \mathrm{~d}\end{array}$} & Yes & 1 & 1.424 & 1.424 & 2 \\
\hline & No & $0=W_{1 R E F}$ & & 0 & 0 \\
\hline \multirow[t]{2}{*}{$\mathrm{CRP} \geq 20.94 \mathrm{mg} / \mathrm{L}$} & Yes & 1 & 2.253 & 2.253 & 3 \\
\hline & No & $0=W_{2 R E F}$ & & 0 & 0 \\
\hline \multirow[t]{2}{*}{$\mathrm{LDH} \geq 461.5 \mathrm{U} / \mathrm{L}$} & Yes & 1 & 1.246 & 1.246 & 2 \\
\hline & No & $0=W_{3 R E F}$ & & 0 & 0 \\
\hline \multirow[t]{2}{*}{ Fever } & Yes & 1 & 1.223 & 1.223 & 2 \\
\hline & No & $0=W_{4 R E F}$ & & 0 & 0 \\
\hline \multirow[t]{2}{*}{ Pleural effusion } & Yes & 1 & 0.841 & 0.841 & 1 \\
\hline & No & $0=W_{5 \text { REF }}$ & & 0 & 0 \\
\hline \multicolumn{6}{|c|}{$\begin{array}{l}\text { Note: 'Yes' represents the basic category, and 'No' represents the other categories. Fixed constant } B= \\
0.841 ; \beta i, \text { regression coefficients of independent risk factors in multivariate logistic regression } \\
\text { equation; } W_{i R E F} \text { risk reference factors; } W_{i j} \text { continuous variables needed to determine their critical } \\
\text { value-i.e., the value corresponding to the maximal Youden index (sensitivity }+ \text { specificity }-1 \text { ) of the } \\
\text { risk factor for each continuous variable-and we then further transformed the value into a categorical } \\
\text { variable and assigned a value ( } \geq \text { critical value was recorded as } 1,<\text { critical value was recorded as } 0) . \\
=1, \ldots, n, \text { where } n \text { is the number of risk factors; } j=1, \ldots, m, \text { where } m \text { is the number of categories; } \beta_{i}\left(W_{i j}\right. \\
\left.-W_{i} \text { REF }\right) \text {, the distance between the category for each risk factor and the basic category. }\end{array}$} \\
\hline
\end{tabular}

According to the score, 395 children with lobar pneumonia caused by MP infection who also underwent bronchoscopic intervention (groups A and B) were divided into high-risk (7-10 points), medium-risk (4-6 points), and low-risk groups ( $0-3$ points): there were 129 cases in the high-risk group, 146 cases in the medium-risk group, and 120 cases in the low-risk group; and 117 cases $(90.71 \%)$ in the high-risk group, 92 cases $(63.01 \%)$ in the medium-risk group, and 20 cases $(16.67 \%)$ in the low-risk group with absolute indications for bronchoscopy. When the scores for children with lobar pneumonia caused by MP infection reached $6,7,8,9$, or 10 points, the possibilities for bronchoscopic intervention were $83.78 \%, 87.69 \%$, $88.33 \%, 97.22 \%$, and $100 \%$, respectively (Fig. 2 ).

\section{Discussion}


MP has received increasing attention in recent years as the principal pathogen causing lobar pneumonia in children. When the effect of conventional anti-infective treatment is not favorable, bronchoscopic intervention therapy for lobar pneumonia caused by MP infection attains increased clinical application. Through this study, we found that children with lobar pneumonia caused by MP infection who demonstrated an absolute indication for bronchoscopy exhibited more severe clinical manifestations and that children without absolute indications for bronchoscopy achieved a better prognosis even without bronchoscopic intervention. Therefore, not all children with lobar pneumonia caused by MP infection should receive bronchoscopic intervention as treatment.

In order to avoid over- or under-treatment in clinical practice, the indications for bronchoscopic treatment of lobar pneumonia caused by MP infection need to be rigorously understood. Therefore, we used binary logistic regression analysis to screen out the independent risk factors for lobar pneumonia caused by MP infection that required respiratory endoscopic intervention and then established an early-warning model.

Our results showed that fever duration before bronchoscopy $\geq 6.5 \mathrm{~d}, \mathrm{CRP} \geq 20.94 \mathrm{mg} / \mathrm{L}, \mathrm{LDH} \geq 461.5$ $U / L$, fever, and complication with pleural effusion were the risk factors for bronchoscopic intervention in children with MP-infected lobar pneumonia. After MP infects the body, it stimulates a series of heat sources and causes the body to continue to generate fever/high fever. Severe MP infection can cause damage to the mucosal-ciliated columnar epithelium of the airways and can even produce shedding. High secretion of airway mucus then leads to the destruction of mucosal-ciliary system function and impairs airway clearance. Airway secretions provide an excellent culture medium for bacteria and viruses, which can easily lead to mixed infections (9). Mixed infections can then aggravate the systemic inflammatory response, heat-peak height, fever time, and hospital stay in children with lobar pneumonia caused by MP infection (10-13). CRP is an acute phase, non-specific reactive protein that is significantly augmented during tissue injury or infection, and studies have shown that CRP can be significantly elevated when MP infection is combined with lung consolidation and pleural effusion (14). The levels of CRP and LDH are also positively correlated with the severity of MP infection (15-17), and MP infection is the primary cause of pleural effusion in children (18-20). After MP infection, specific antibodies induce an autoantibody reaction, pleural disease, and finally fibrinous exudates, which often represent a stronger immune response.

Studies have shown that age, duration of fever, CRP, and LDH are independent risk factors for intrabronchial mucus in children with MPP (21); when children with MPP have CRP $\geq 12.27 \mathrm{mg} / \mathrm{L}$ and $\mathrm{LDH} \geq 462.65 \mathrm{U} / \mathrm{L}$, there is a possibility of the formation of mucus plugs in the bronchus (22). Other studies have shown that $C R P>44 \mathrm{mg} / \mathrm{L}$ and $\mathrm{LDH}>480 \mathrm{U} / \mathrm{L}$ are independent risk factors for children with refractory MPP undergoing multiple bronchoscopic interventions. In one scoring system for refractory MPP in children undergoing multiple bronchoscopic interventions, CRP > 44 mg/L and LDH > 480 U/L were assigned 1 point each (23). In this study, we established an early-warning model of bronchoscopic intervention treatment for children with lobar pneumonia caused by MP infection in which CRP $\geq 20.94$ $\mathrm{mg} / \mathrm{L}$ elicited 3 points, fever duration before bronchoscopy $\geq 6.5 \mathrm{~d}$ received 2 points, $\mathrm{LDH} \geq 461.5 \mathrm{U} / \mathrm{L}$ received 2 points, fever received 2 points, and complication with pleural effusion received 1 point, for a 
total score of 10 points. When the score was $\geq 6$, the possibility of bronchoscopic intervention was $>80 \%$, and the higher the score, the greater the probability of bronchoscopic intervention.

This study possessed some limitations. First, this was a retrospective study, and the selected research subjects may have shown selection bias. Factors such as the long research timespan, the limitations of the test levels, and the geographical limitations also exerted certain influences on the establishment of the predictive model. Second, the early-warning model established by our research is still only in the initial stage, and it needs to be further tested and improved in the clinic to provide clinicians with a simple, convenient, and valuable scoring system-which should then be beneficial in clinical treatment.

In conclusion, we herein presented an early-warning model of respiratory endoscopic intervention in children with lobar pneumonia caused by Mycoplasma pneumoniae infection and showed that the evaluative effect of the early-warning model was acceptable (with an AUC for the predictive score of 0.859) - with suitable accuracy and clinical practicability.

\section{Abbreviations}

MPP: Mycoplasma Pneumoniae Pneumonia WBC: White blood cells;ALT: Alanine aminotransferase; AST: Aspartate amino transferase; CAP: Community-acquired pneumonia; MRMP macrolide-resistant Mycoplasma pneumoniae CARDS Tx community-acquired respiratory distress syndrometoxin BALF Bronchoalveolar lavage fluid NPA nasopharyngeal aspirates CD: Cluster of differentiation; Cl: Confidence interval; CRP: C reactive protein; CS: Corticosteroid;CT: Computed tomography; DD: D-dimer; EOS: Eosinophil count;IgA: Immunoglobulin A; IgG: Immunoglobulin G;LDH: Lactate dehydrogenase; NPA: Nasopharyngeal aspirates; OR: Odds ratio; ROC: Receiver operating characteristic; AUC: Areaunder curve; BMP: Bronchial mucus plugs; PA, prealbumin; PCR, polymerase chain reaction

\section{Declarations}

Ethics approval and consent to participate

This study protocol was approved by the Ethical Review Committee of Children's Hospital of Soochow University with judgment's reference number 2020CS078. Authors reporting data collection from humans, interviews confirm that all experiments were performed in accordance with relevant guidelines and regulations. Informed consent was obtained from all their legal guardian(s) before data collection.A statement to confirm that all methods were carried out in accordance with relevant guidelines and regulations.

Availability of data and materials

All data generated or analysed during this study are included in this published article. 
Competing interests

The authors declare that they have no competing interests.

Funding

This work was supported by grants from the following projects: Social Development Projects of Jiangsu Province (grant NO. BE2019671); Science and Technology Program of Suzhou (grant NO. SS201869); the National Natural Science Foundation of China (grant NO. 81970027; 81870006;81771676; 81971490); Jiangsu Provincial Medical Youth Talent (grant NO. QNRC2016766); Suzhou Medical Youth Talent (grant NO. GSWS2019047); Key Lab of Respiratory Disease of Suzhou (grant NO. SZS201714); Suzhou Medical Technology Projects of Clinical Key Diseases (grant NO. LCZX201809); The Postgraduate Research \& Practice Innovation Program of Jiangsu Province (grant NO. KYCX20 2727). The funders had no role in study design, data collection and analysis, decision to publish, or preparation of the manuscript. This work was supported by the following projects.

Authors' contributions

All authors have read and approved the manuscript. JZ and ZC conceivedand designed the study. LH, TW and YM made contributions to the analysisand interpretation of data. YY collected the clinical data. ZS contributed to the statistical analysis.

Acknowledgements

We thank LetPub (www.letpub.com) for its linguistic assistance during the preparation.

Consent for publication

Not applicable.

\section{References}

1. Chen Z, Shang X, Zhao S, et al. Expert Consensus on Diagnosis and Treatment of Mycoplasma Pneumoniae Pneumonia in Children. Chin J Appl Clin Pediatr (2015) 30:1304-8.

2. Wang BJ, Zhang H. Research Progress of Macrolide-Resistant Mycoplasma Pneumoniae. Lab Med (2020) 35:1070-4.

3. Ma XL, Ding ZB, Huang YK. Macrolide-Resistant Mycoplasma Pneumoniae Pneumonia in Children: Current Status and Progress in Mechanism Studies. J Int Pharm Res (2020) 47:804-8.

4. Zhang H, Shang YX. Value of Fiberoptic Bronchoscopy in the Diagnosis and Treatment of Refractory Mycoplasma Pneumoniae Pneumonia in Children. Chin J Pract Pediatr (2019) 34:504-7.

5. Li SS, Zhang W, Wang YM, Gu Q. Effect of Fibro Bronchoscopy in Diagnosis and Treatment of Different Course of Lobar Pneumonia in Children. Matern Child Health J Care China (2015) 30:3194- 
6.

6. Jiang ZF, Shen KL, Shen Y, et al. Zhu Futang Ractice of Pediatric. 8 edition. Beijing: China Academic Journal Electronic Publishing House (2015). p. 1253-95.

7. Zhong LL, Men FZ, Men C, et al. Expert Consensus on the Diagnosis and Treatment of Respiratory Endoscopic Intervention in Children with Refractory Pneumonia in China. Chin J Pract Pediatr (2019) 34:449-57.

8. Lin YH, Bai J, Pan ZJ, et al. Scoring Method to Evaluate the Effect of Bronchoscopy Alveolar Lavage in the Treatment of Lobar Pneumonia in Children. Zhejiang Clin Med (2018) 20:831-3.

9. Liu JR, Zhao SY. Mechanisms of Refractory Mycoplasma Pneumoniae Pneumonia in Children. Int J Clin Pediatr (2013) 31:1186-8.

10. Lu YH, Zhang XX, Yan YD, et al. Role and Influence of Coinfection Factor to Refractory Mycoplasma Pneumoniae Pneumonia in Children. Int J Clin Pediatr (2017) 35:81-5.

11. Mao LY (2018). Clinical Study on Mixed Infection of Mycoplasma Pneumonia in Hospitalized Children. [master's thesis]. [Suzhou]: Soochow University

12. Ye H, Zhang CL, Liu X, et al. Analysis on Mixed Infections in Children with Mycoplasma Pneumoniae Pneumonia. Medical Recapitul (2013) 19:2456-8.

13. Chen LL (2013). Study on Mixed Infection of Mycoplasma Pneumoniae Pneumonia in Children. [master's thesis]. [Hangzhou]: Zhejiang University

14. Hsieh CC, Tang RB, Tsai CH, Chen W. Serum Interleukin-6 and Tumor Necrosis Factor-Alpha Concentrations in Children with Mycoplasma Pneumonia. J Microbiol Immunol Infect (2001) 34:109-12.

15. Mei YX, Jiang JJ, Cai B, et al. Clinical Risk Factor Analysis of Childhood Refractory Mycoplasma Pneumoniae Pneumonia. Int J Clin Pediatr (2014):1138-40.

16. Seo YH, Kim JS, Seo SC, Seo WH, Yoo Y, Song DJ, et al. Predictive Value of C-Reactive Protein in Response to Macrolides in Children with Macrolide-Resistant Mycoplasma Pneumoniae Pneumonia. Korean J Pediatr (2014) 57:186-92. doi: 10.3345/kjp.2014.57.4.186

17. Izumikawa K, Izumikawa K, Takazono T, Kosai K, Morinaga Y, Nakamura S, et al. Clinical Features, Risk Factors and Treatment of Fulminant Mycoplasma Pneumoniae Pneumonia: A Review of the Japanese Literature. J Infect Chemother (2014) 20:181-5. doi: 10.1016/j.jiac.2013.09.009

18. Zhang Y, Cao L, Zhu CM, et al. Analysis of the Etiology and Clinical Characteristics of 177 Children with Pleural Effusion. Chin J Pract Pediatr (2017) 32:227-9.

19. Nie YQ. Analysis of Etiology and Clinical Characteristics of 56 Children with Pleural Effusion. World Compd Med (2019) 5:47-9.

20. Zhang XX, Ji W, Gu WJ, et al. Analysis of Etiological Characteristics of Pneumonia Complicated with Pleural Effusion in Hospitalized Children. J Pediatr Pharm (2020) 26:14-8.

21. Xu Q, Zhang L, Hao C, et al. Prediction of Bronchial Mucus Plugs Formation in Patients with Refractory Mycoplasma Pneumoniae Pneumonia. J Trop Pediatr (2017) 63:148-54. 
22. Zhang J, Wang T, Li R, et al. Prediction of Risk Factors of Bronchial Mucus Plugs in Children with Mycoplasma Pneumoniae Pneumonia. BMC Infect Dis (2021) 21:67.

23. Zhang R (2018). Risk Factors and Predictive Scoring System for Multiple Bronchoscope Adjuvant Therapy in Children with Refractory Mycoplasma Pneumoniae Pneumonia. [master's thesis]. [Suzhou]: Soochow University

\section{Figures}

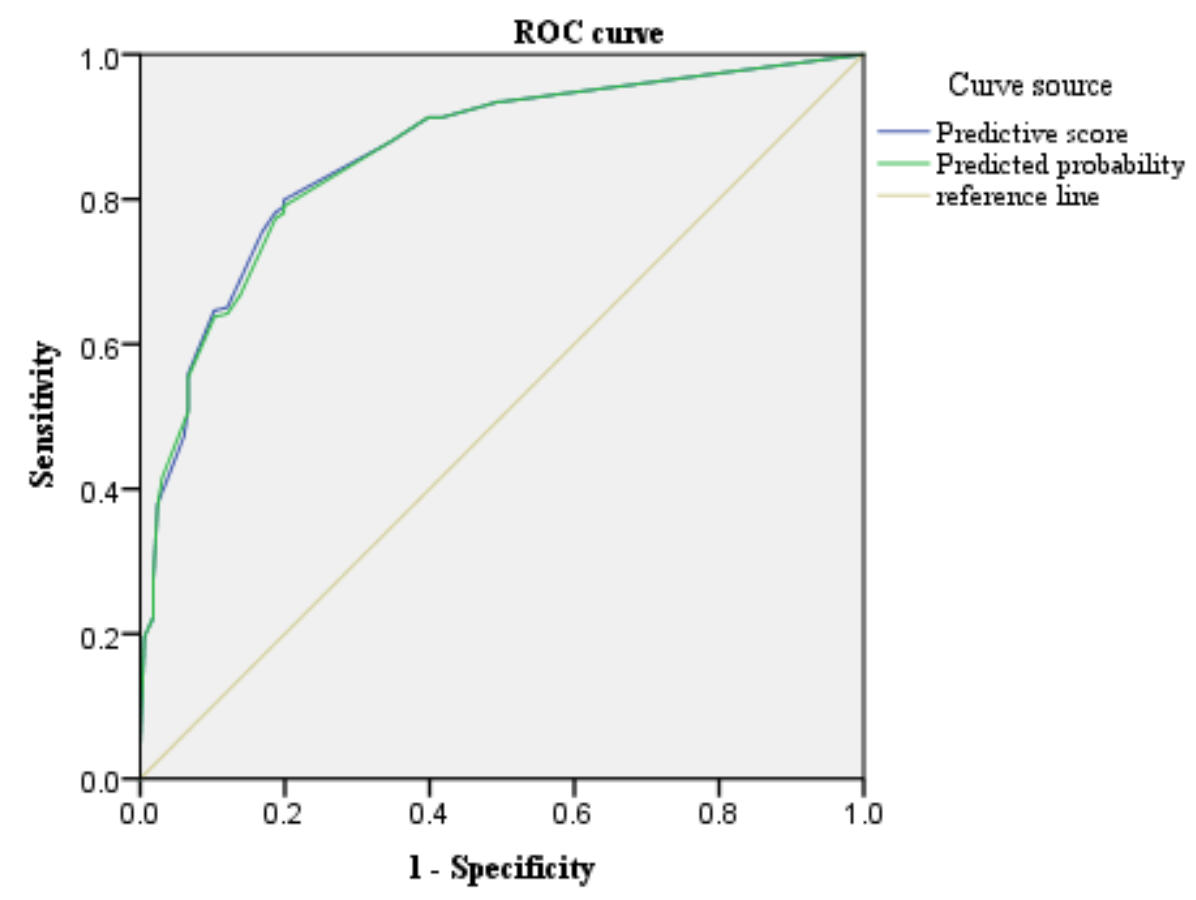

\section{Figure 1}

Regression model and receiver operating characteristic (ROC) curve of predictive score. 
120

\section{Percentage}

100

80

60

40

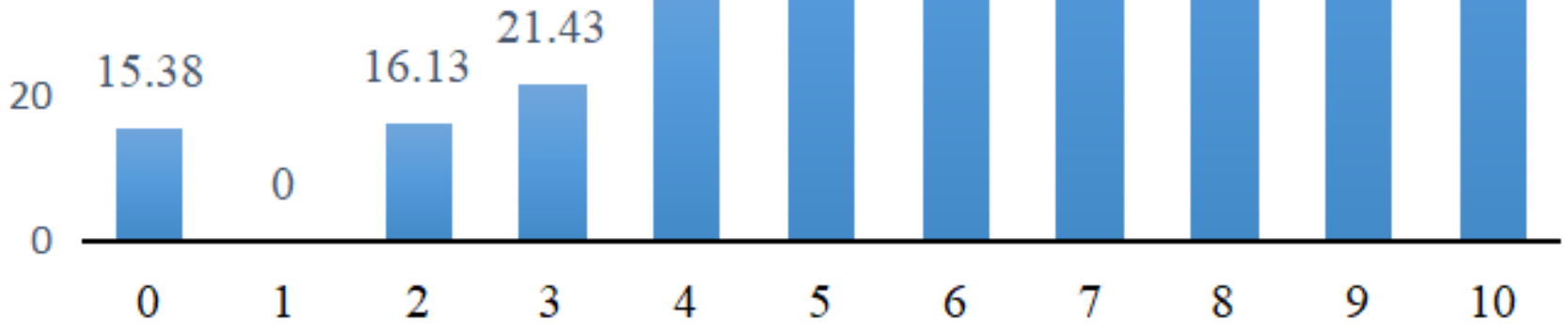

\section{Figure 2}

Percentage of children with lobar pneumonia caused by Mycoplasma pneumoniae (MP) infection in each scoring group who required bronchoscopic intervention. 